\title{
3D Face Texture Modeling from Uncalibrated Frontal and Profile Images
}

\author{
Hu Han and Anil K. Jain \\ Department of Computer Science and Engineering \\ Michigan State University, East Lansing, MI, U.S.A. \\ $\{$ hhan, jain\}@cse.msu.edu
}

\begin{abstract}
$3 D$ face modeling from $2 D$ face images is of significant importance for face analysis, animation and recognition. Previous research on this topic mainly focused on $3 D$ face modeling from a single $2 D$ face image; however, a single face image can only provide a limited description of a $3 D$ face. In many applications, for example, law enforcement, multi-view face images are usually captured for a subject during enrollment, which makes it desirable to build a $3 D$ face texture model, given a pair of frontal and profile face images. We first determine the correspondence between uncalibrated frontal and profile face images through facial landmark alignment. An initial $3 D$ face shape is then reconstructed from the frontal face image, followed by shape refinement utilizing the depth information provided by the profile image. Finally, face texture is extracted by mapping the frontal face image on the recovered $3 D$ face shape. The proposed method is utilized for $2 D$ face recognition in two scenarios: (i) normalization of probe image, and (ii) enhancing the representation capability of gallery set. Experimental results comparing the proposed method with a state-of-the-art commercial face matcher and densely sampled LBP on a subset of the FERET database show the effectiveness of the proposed $3 D$ face texture model.
\end{abstract}

\section{Introduction}

Automatic face recognition is a challenging task that has gained substantial attention during the last three decades [22]. Face Recognition Vendor Test (FRVT) [27, 29] evaluated state-of-the-art face recognition systems through a series of large scale independent evaluations in both constrained and semi-constrained environments. The results indicated that many $2 \mathrm{D}$ face recognition systems can achieve acceptable performance in constrained environments (e.g. frontal pose, indoor illumination and natural expression). However, FRVT evaluations also revealed that face recognition in unconstrained environments (e.g. large facial appearance variations due to head pose, non-uniform light- ing conditions, occlusion, and facial expressions) is still far from satisfactory. One of the main reasons for this limited performance in unconstrained situations is that face image is only a $2 \mathrm{D}$ projection of a $3 \mathrm{D}$ human face, which is very sensitive to the facial appearance variations. This motivates the use of 3D facial shape and texture in face recognition.

The most direct method to perform 3D face recognition is to capture 3D face images (shape and texture) using 3D sensors both in constructing the probe and galley sets $[21,24]$. However, 3D sensing is still relatively quite expensive and the acquisition time can be slow. These factors make it infeasible to utilize 3D scanners in many practical applications. This means that most deployed face recognition systems can only perform recognition based on 2D face images. To resolve this conflict between the unavailability of 3D face data and the requirement of improving recognition performance in unconstrained environments, two different strategies have been proposed: (i) normalization of 2D face images, and (ii) 3D face modeling from 2D images.

The first approach above normalizes facial appearance variations by performing a number of different transformations in 2D image space. For example, illumination normalization methods $[30,38,13,15,34,19,33,31,14]$ are applied to eliminate uneven lighting variations, and pose normalization methods $[7,10,11,37,36]$ are applied to generate a virtual frontal view from an arbitrary non-frontal view. However, each normalization method can only handle a specific kind of facial appearance variation. Thus, normalization of 2D face images can improve the face recognition performance when there is only one major facial appearance variation. Moreover, some normalization methods for 2D face images assume that lighting directions or face poses are known. These constraints limit the application of normalization methods for $2 \mathrm{D}$ face images in practice, as the factors (pose, illumination and expression) leading to facial appearance variation are usually not known a priori.

The feasibility of building a 3D face model from one or more $2 \mathrm{D}$ face images makes it possible to address various challenges due to unknown facial appearance variations. Directly applying Shape-from-Shading (SFS) [17] 


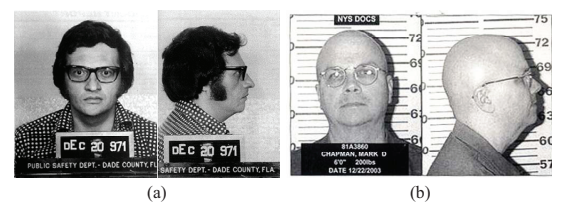

Figure 1. Examples of mug shot face images in frontal and profile views acquired at the booking time by police and law enforcement agencies. (a) Mug shots of a suspect who was arrested in 1971 in Miami, and charged with grand larceny ${ }^{1}$. (b) Mug shots of a suspect who was arrested for a murder in December $1980^{2}$.

to a single $2 \mathrm{D}$ image for face analysis is found to be very difficult, since the concave-convex ambiguity may lead to inversion of facial components [9]. To address this problem, one common approach is to impose domain specific constraints or use a face-specific shape model. By exploiting the approximate bilateral symmetry of faces, symmetric SFS $[42,43]$ was proposed to recover both shape and albedo information from a single face image. A constraint on 3D face shape was incorporated in SFS [5] by deriving a low dimensional parameterization of face shape space. Based on principal component analysis (PCA) of 3D head shapes and texture captured with 3D scanners, a 3D morphable model [8] was proposed to simultaneously estimate 3D face shape, texture, and pose as well as lighting and camera properties from one single face image. By replacing the texture fitting in the original 3D morphable model with 2D image mapping, an efficient method was proposed for 3D face reconstruction from one frontal face image [20]. 3D morphable model was combined with spherical harmonics model [6] to recover the face shape, texture, and lighting information from a single image [39].

The above approaches focus on 3D face modeling from a single $2 \mathrm{D}$ face image, which is desirable for face recognition tasks when only one face image is available for each individual. However, with the rapid development of digital cameras, multi-view face images and even video sequences of a subject can be captured in order to achieve more accurate recognition performance. For example, mug shot databases managed by law enforcement agencies generally include two mug shots; one for frontal face and the other for profile face (see Fig. 1). The reason for using these two face images lies in the fact that the information provided by a profile image is complementary to that provided by a frontal image. As shown in Fig. 2, the frontal face image provides the most descriptive information of a 3D face in the $X-Y$ plane, while the profile face image provides the most descriptive information of a 3D face in the $Y-Z$ plane. In these applications, 3D face modeling methods should utilize information provided by both the frontal and

\footnotetext{
${ }^{1}$ http://cobbloviate.com/2010/03/is-a-mug-shot-worth-more-than-athousand-words.html

${ }^{2} \mathrm{http}: / /$ strangecosmos.com/content/item/102888.html
}

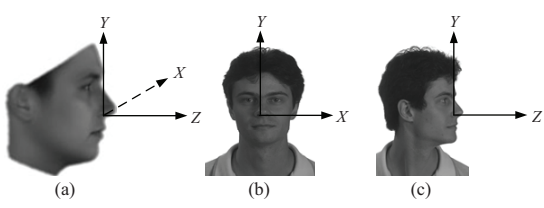

Figure 2. Different types of information provided by frontal and profile face images. (a) A three dimensional coordinate system defined for a 3D human face. (b) A frontal face image mainly provides description of a 3D face within the $X-Y$ plane. (c) A profile face image mainly provides description of a 3D face within the $Y-Z$ plane (depth information).

profile face images. However, compared to 3D face modeling from a single face image, 3D face modeling from frontal and profile face images has not received wide attention.

In this paper, we present a method for 3D face texture modeling from frontal and profile face images. Since the frontal and profile face images are usually captured without calibration, we first align the shapes in frontal and profile face images through landmark alignment. An initial 3D face shape is reconstructed from the frontal face image, followed by $3 \mathrm{D}$ shape refinement utilizing the profile face image. Finally, facial texture is extracted by mapping the frontal face image to the recovered 3D face shape. The effectiveness of the proposed approach is verified by face images synthesized for novel views as well as face recognition across pose on a multi-view subset of the FERET [28] database.

\section{Related Work}

Compared to 3D face modeling from a single face image, only a limited amount of research is available in the literature on 3D face modeling from uncalibrated frontal and profile face images. Ip and Yin [18] developed a scheme for automatically constructing a 3D individualized head model from two orthogonal views (actually a side view and the frontal view of the face). In their approach, facial landmarks on a $2 \mathrm{D}$ face image were used to deform the corresponding vertices in a generic model; the remaining vertices in the generic model were calculated via interpolation. The two input facial images were then blended and texture-mapped onto the 3D head model to get the face texture. Ansari and Mottaleb [4] also built a 3D shape model through interpolation based on the $3 \mathrm{D}$ coordinates of 15 vertices that are calculated from two calibrated face images in orthogonal views. As pointed out by the authors, their method could not be applied to uncalibrated frontal and profile face images in public face databases. Heo and Savvides [16] first estimated a sparse 3D face shape by aligning a frontal and a profile face image. The final 3D face shape was also calculated using an interpolation scheme (cubic spline interpolation). The face texture was extracted by mapping the frontal face image to the calculated 3D face shape. The effectiveness of these approaches was shown by present- 


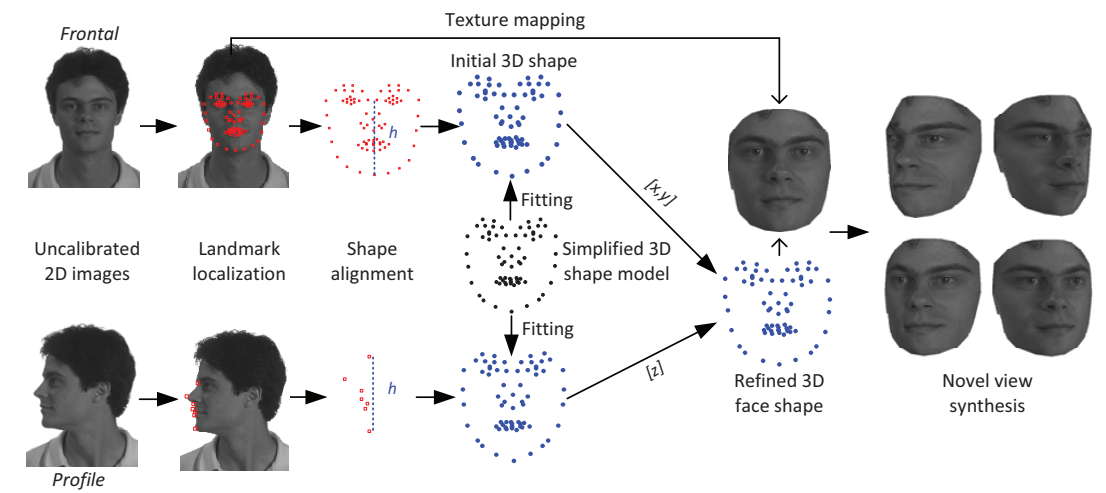

Figure 3. Overview of the proposed 3D face texture modeling method from uncalibrated frontal and profile face images.

ing visually appealing 2D face images synthesized for novel views. However, no face recognition experiments were performed on public face databases containing multi-view face images. Thus, the effectiveness of their approaches is unknown for face recognition. Zhang et al. [40] generated the $3 \mathrm{D}$ face shape by proposing a multilevel variation minimization approach and extracted the face texture by taking diffuse and specular reflections of face surface into account. They evaluated their approach by both virtual view synthesis and face recognition experiments. However, face recognition was performed on a small-scale database (CMU-PIE [32]), which contains only 68 subjects. Moreover, only eigenfaces [35] and local binary patterns (LBP) [25] were utilized in the face matching algorithms. The effectiveness of their 3D modeling approach in conjunction with state-ofthe-art face matchers is not known.

Besides 3D face modeling from two face images, 3D face modeling has also been extended to multiple $2 \mathrm{D}$ face images. Zhao et al. [41] estimated the 3D face shape by using frontal and profile 2D morphable shape models. The face texture was calculated as a weighted combination of multiple input face images. Again, only face images synthesized for novel views were shown. Faggian et al. [12] presented a mathematical derivation for 3D face modeling algorithm from one single face image and made it possible to be directly applied to multiple $2 \mathrm{D}$ face images. The accuracy of the reconstructed 3D face shape was verified through cross-validation on USF Human ID 3D database [3]. Again, no experiments were performed to verify the effectiveness of their 3D face modeling method for face recognition. Moreover, in both the above methods, there is no comparison showing that the 3D face model built from multiple face images is more accurate than that built from only frontal and profile face images.

\section{3D Face Texture Modeling}

Considering that a pair of frontal and profile face images should be able to provide the most essential description of a 3D human face, we propose to build a 3D face texture model for each subject from uncalibrated frontal and profile face images. To recover the 3D face shape, we employ a statistical model considering its robustness, instead of the commonly used vertex interpolation based on 3D face vertices. To achieve efficient 3D modeling, the face texture is extracted by directly mapping the frontal face image to the recovered 3D face shape. As illustrated in Fig. 3, the proposed 3D face texture model consists of the following steps:

1) Landmark localization for face images.

2) Shape alignment between frontal and profile face images.

3) Initial 3D shape reconstruction from frontal image.

4) 3D shape optimization based on profile face image.

5) Texture extraction by mapping frontal face image according to the recovered $3 \mathrm{D}$ face shape.

\subsection{Landmark Localization}

Since the frontal and profile face images are uncalibrated, we need to establish the correspondence between them. For the proposed approach, a sparse correspondence between the frontal and profile face images is sufficient. Thus, we determine the correspondence by localizing predefined feature points on both frontal and profile face images. For frontal face images, an Active Shape Model (ASM) [10] is utilized to detect a set of facial landmarks. Specifically, the open source software Stasm [23] is utilized as the ASM detector. To further improve the robustness of Stasm, we initialize Stasm by providing the eye locations detected by PittPatt Face Recognition SDK [2]. For the profile face images in the gallery set, we manually mark several facial landmarks. Since the main goal of 3D face texture modeling from frontal and profile face images is to achieve higher recognition performance in unconstrained environments of value to law enforcement agencies, it is reasonable 


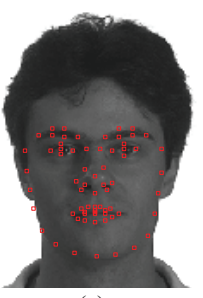

(a)

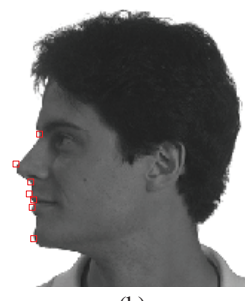

(b)
Figure 4. Facial landmark localization. (a) Automatically detected facial landmarks for a frontal face image. (b) Manually marked facial landmarks for a profile face image.

to allow manual landmark annotation in order to build more accurate 3D face texture model. For example, to determine the identity of a suspect in a criminal investigation, it is acceptable to manually mark the facial landmarks, particularly in low quality video frames. Fig. 4 illustrates the localized facial landmarks for a pair of frontal and profile face images. Fig. 4 (a) shows the 68 automatically detected facial landmarks using ASM, and Fig. 4 (b) shows the 7 manually annotated landmarks on a profile face image.

\subsection{Correspondence Determination}

Once we have the facial landmarks on both frontal and profile face images, the correspondence between the two uncalibrated face images can be determined through landmark alignment. We denote the landmarks on a frontal face image as $\left(x_{i}^{f}, y_{i}^{f}\right), i=1,2, \cdots, t$ (here $\left.t=68\right)$ and those on a profile face image as $\left(x_{j}^{p}, y_{j}^{p}\right), j=1,2, \cdots, t^{\prime}$ (here $t^{\prime}=7$ ). Note that although the landmarks on frontal and profile face images are of the same form, they have different physical meaning. The coordinates $x_{j}^{p}$ and $y_{j}^{p}$ in profile face image correspond to the $Z$ and $Y$ axes, respectively in the 3D face model. This suggests that landmark alignment should be conducted based on the $\mathrm{Y}$ coordinates. From Fig. 2, we can also notice that $Y$-axis is the common axis between the frontal and profile face images.

Based on the analysis above, two control points, $C_{1}$ and $C_{2}$, are utilized for landmark alignment. As illustrated in Fig. 5 (a), $C_{1}$ is the midpoint between the two eyes and $C_{2}$ is the landmark on the chin (landmark \#8). Based on control points $C_{1}$ and $C_{2}$, the frontal and profile face images are, respectively, rotated and scaled such that the direction of the line connecting $C_{1}$ and $C_{2}$ is vertical and the length of the line equals that in the average 3D face shape. Moreover, we convert the coordinates of both vertices in $3 \mathrm{D}$ face shape and landmarks in 2D face image by using point $O$ (landmark \#42 in Fig. 5 (a)) as the origin. This conversion makes the coordinate system independent of the face image size.

After the above landmark alignment, the correspondence between the uncalibrated frontal and profile faces is determined. Another benefit of landmark alignment is that it sim-

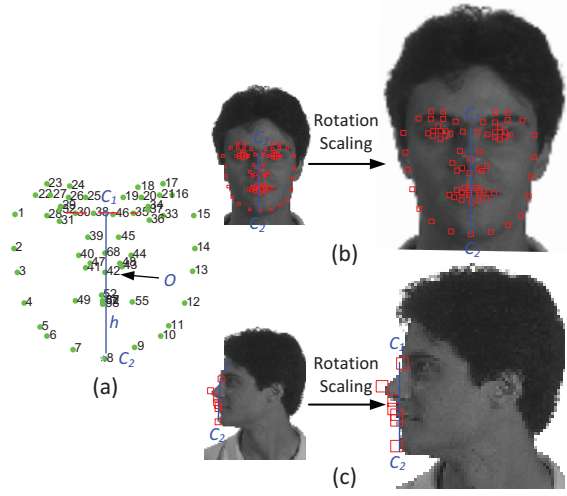

Figure 5. Landmark alignment for frontal and profile face images. (a) Two control points, $C_{1}$ and $C_{2}$, used for landmark alignment are illustrated on an average $3 \mathrm{D}$ face shape ${ }^{1}$. A frontal face image in (b) and a profile face image in (c) are rotated and scaled such that the direction of the line connecting $C_{1}$ and $C_{2}$ is vertical and the length of the line equals that in the average $3 \mathrm{D}$ face shape.

plifies the procedure for fitting the average 3D face shape to a 2D face image, which will be discussed in the following.

\subsection{Initial 3D Shape Reconstruction}

As discussed in Section 2, an interpolation based algorithm is used to recover the 3D face shape, after the correspondence between the frontal and profile face images is determined. Different from previous work, we employ a statistical model to reconstruct the 3D face shape, because of its robustness.

Specifically, we use a simplified 3D morphable model [8] without the texture fitting. We derive our 3D shape model from the USF Human ID 3-D Database [3], which includes 3D face shape and texture of 100 (denoted by $m$ ) subjects captured with a 3D scanner. Each 3D face includes 75,972 vertices which are interactively reduced to $n$ (here, $n=t=68$ ) for efficient performance [26]. We then represent each $3 \mathrm{D}$ shape with a shape vector $\mathbf{S}=$ $\left(x_{1}, y_{1}, z_{1} ; x_{2}, y_{2}, z_{2} ; \cdots ; x_{n}, y_{n}, z_{n}\right)^{T}$, which is a concatenation of the $X, Y, Z$ coordinates of the $n$ vertices. PCA is then applied to all $m$ shape vectors ( $m$ is the number of subjects) to get a statistical model of 3D face shape

$$
\mathbf{S}^{\prime}=\overline{\mathbf{S}}+\sum_{k=1}^{K} \alpha_{k} \mathbf{W}_{k}
$$

where $\mathbf{S}^{\prime}$ is a new $3 \mathrm{D}$ face shape; $\overline{\mathbf{S}}$ is the average $3 \mathrm{D}$ face shape; $\mathbf{W}_{k}$ is the eigenvector of all $m$ 3D face shapes that corresponds to the $k$-th largest eigenvalue; $\alpha_{k}$ is the set of coefficients for the $k$-th shape eigenvector. Given a set of facial landmarks $\mathbf{P}_{2 D}=\left(x_{1}, y_{1} ; x_{2}, y_{2} ; \cdots ; x_{t}, y_{t}\right)^{T}$ for

\footnotetext{
${ }^{1}$ Average 3D face shape is calculated by averaging 100 3D shapes in the USF Human ID 3D database [3].
} 
a frontal 2D face image, the 3D shape for this image can be efficiently estimated by minimizing the following cost function using an iterative algorithm proposed in [26]

$$
e\left(\mathbf{P}, \mathbf{R}, \mathbf{T}, s,\left\{\alpha_{k}\right\}_{k=1}^{K}\right)=\left\|\mathbf{P}_{2 D}-s \cdot \mathbf{P R T S}^{\prime}\right\|_{L_{2}}
$$

where $\mathbf{T}, \mathbf{R}, s$ and $\mathbf{P}$ are the translation, rotation, and scaling operations for the 3D face shape $\mathbf{S}^{\prime} . \mathbf{P}$ is an orthogonal projection from $3 \mathrm{D}$ to $2 \mathrm{D}$.

As mentioned in Section 3.2, the landmark alignment also facilitates the process of fitting the generic 3D face to $2 \mathrm{D}$ face images. The reason is threefold. First, since $\mathbf{P}_{2 D}$ and $\overline{\mathbf{S}}$ have the same coordinate origin, the translation operation $\mathbf{T}$, can be omitted. Secondly, $\mathbf{P}_{2 D}$ and $\overline{\mathbf{S}}$ are already of the same scale after the landmark alignment, which means parameter $s=1$ and scaling operation can also be omitted. Thirdly, unless there is a dramatic pitch rotation, the rotation operation $\mathbf{R}$ for fitting a 3D shape model to a frontal face image can be omitted and the rotation for fitting a 3D shape model to a profile face image can be simplified as a 90 degree yaw rotation. Therefore, we mainly need to estimate the PCA coefficients $\left\{\alpha_{k}\right\}_{k=1}^{K}$. With the above statistical model, we first reconstruct an initial 3D face shape $\mathbf{S}_{f}$ from the frontal face image.

\subsection{D Shape Refinement}

As illustrated in Fig. 2, the frontal face image provides a detailed description for a 3D face in the $X-Y$ plane; thus the $X-Y$ coordinates of the semantic feature points in the initial 3D face shape should be accurately estimated. However, the $Z$ coordinates of the initial 3D face shape $\mathbf{S}_{f}$ are mainly derived from the average $3 \mathrm{D}$ face shape $\overline{\mathbf{S}}$. Therefore, the initial 3D face shape $\mathbf{S}_{f}$ should be refined by improving the accuracy of the $Z$ coordinates.

To calculate the $Z$ coordinates of 3D face shape from a profile face image, we further reduce 3D face shape model in Section 3.3 from $n$ vertices to $n^{\prime}$ (here, $n^{\prime}=t^{\prime}=7$ ) vertices, and fit it to the $2 \mathrm{D}$ facial landmarks on a profile face image. This way, we get another $3 D$ face shape $\mathbf{S}_{p}^{\prime}$ and the coefficients $\alpha_{k}^{\prime}, k=1,2, \cdots, K^{\prime}$ corresponding to the eigenvectors. We should notice that $\mathbf{S}_{p}^{\prime}$ has fewer vertices than the initial 3D face shape $\mathbf{S}_{f}$. Thus, we calculate a "high resolution" 3D face shape $\mathbf{S}_{p}$ based on $\alpha_{k}^{\prime}$

$$
\mathbf{S}_{p}=\overline{\mathbf{S}}+\sum_{k=1}^{K^{\prime}} \alpha_{k}^{\prime} \mathbf{W}_{k}
$$

Now, $\mathbf{S}_{p}$ has the same number of vertices as $\mathbf{S}_{f}$.

Given the landmark alignment in Section 3.2, the reconstructed 3D face shape $\mathbf{S}_{p}$ is in the same coordinate system and of the same scale as $\mathbf{S}_{f}$. Next, we refine the initial 3D face shape $\mathbf{S}_{f}$ with a displacement of its $Z$ coordinates by the $Z$ coordinates of $\mathbf{S}_{p}$. Fig. 6 illustrates the initial and

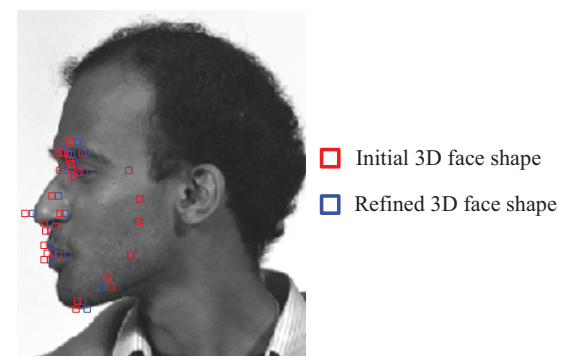

Figure 6. 3D face shape refinement based on a profile face image.

refined 3D face shapes by respectively plotting them on a profile face image using red and blue rectangles. For the facial component with large depth variations (e.g. nose), the refined 3D face shape is more accurate than the initial 3D face shape.

\subsection{Texture Extraction}

To extract the face texture, we directly map the frontal face image to a novel pose based on Delaunay triangulation of the 2D facial landmarks. With this direct mapping, we retain detailed and realistic features in face image, which helps in face recognition.

\section{Experimental Results}

In this section, we study the performance of the proposed 3D face texture model through face recognition across pose. The proposed 3D face texture model is used to improve 2D face recognition accuracy in two ways: (i) face normalization, and (ii) representation capability enhancement. The first application of the model is appropriate for the realworld scenario where law enforcement agencies find its mate in a large scale mug shot database. In this scenario, the proposed 3D face texture model is utilized to synthesize a frontal face image for the probe, which is then matched to the gallery set to determine the identity of the probe image. The second application of our model is suitable in situations where multi-view face images (typically including a pair of frontal and profile face images) are captured for each subject during enrollment for the purpose of highly accurate authentication. In this scenario, the proposed $3 \mathrm{D}$ face texture model is used to synthesize various face images for each subject in the gallery set in order to enhance the representation capability of each class (subject). The above two scenarios also represent two different tasks in face recognition: identification and verification.

The experiments are conducted on a subset of the FERET [28] database, which contains face images with multiple pose variations (see Fig. 7). In order to build the 3D face texture model, a frontal face image (fa) and a profile face image (pl) of each subject are used as the gallery set. To reduce the laborious cost of manual annotation of face images, the facial landmarks for frontal face images are lo- 


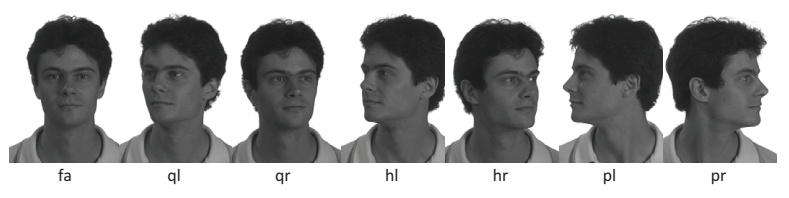

Figure 7. Pose variations in the multi-view subset of the FERET [28] database.

Table 1. Datasets used for the face identification experiment. The proposed method is utilized to normalize the pose of probe images.

\begin{tabular}{cccc}
\hline \multicolumn{2}{c}{ Subset } & \# Subjects & \# Images \\
\hline \multirow{2}{*}{ Gallery set } & FERET & 505 & 1,010 \\
& PCSO & 10,000 & 10,000 \\
\hline Probe set & FERET & 280 & 403 \\
\hline
\end{tabular}

calized automatically using ASM and only seven landmarks are manually annotated for the profile face images. Among face images of the remaining five poses (ql, qr, hl, hr, pr), those for which facial landmarks can be automatically detected using ASM are used as the probe set.

To evaluate the effectiveness of the proposed model, we use densely sampled LBP and a state-of-the-art commercial face matcher FaceVACS [1] for face recognition. The proposed 3D face modeling method can be claimed to be of value if it can be shown to improve the performance of a state-of-the-art face recognition system.

\subsection{Face Normalization}

In the first scenario, the proposed 3D face texture model is used to normalize the probe face images. To replicate real-world applications in law enforcement agencies, the gallery is enlarged by 10,000 mug shot photographs (one image per subject) from the Pinellas County Sheriff's Office (PCSO) database. The dataset configuration for this experiment is shown in Table 1. In this identification task, 403 probe face image with arbitrary pose variation are matched against 11,010 background face images.

Figure 8 shows the the original probe images (the first row) and the normalized probe images (the second row) by the proposed 3D texture model. We can notice that detailed facial features, e.g. mole, are well preserved in the normalized images.

The normalized probe face images are matched to the gallery set by using the commercial matcher FaceVACS and densely sampled LBP. Figure 9 shows the performance of FaceVACS and LBP. FaceVACS and LBP achieve, respectively, $91.6 \%$ and $82.1 \%$ rank-1 recognition rates after probe face images are normalized using the proposed 3D face texture model, which are much better than the performance before face normalization $(83.6 \%$ and $72.5 \%)$. These results demonstrate the effectiveness of the proposed 3D face texture model for 2D face identification.

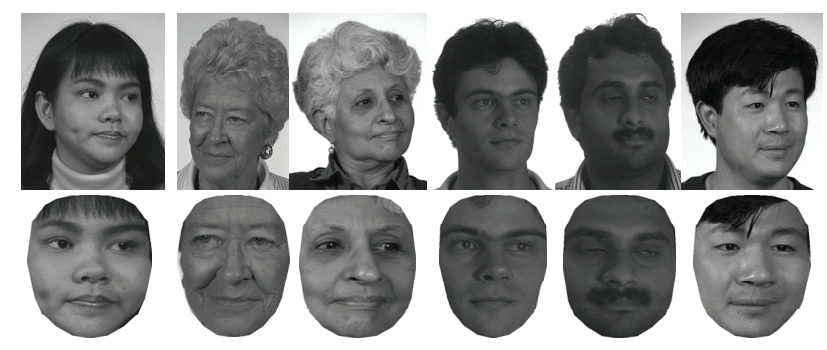

Figure 8 . Normalized probe face images (bottom row) after applying the proposed 3D face texture model to a single input face image (top row).

\subsection{Representation Capability Enhancement}

In the second scenario, for improving the face authentication accuracy, we utilize the frontal and profile face images that are usually captured for each subject during booking time (enrollment). Compared with a single frontal face image, a pair of frontal and profile images provides better representation capability for a subject's face. In this experiment, we propose to enhance the representation capability of each subject with the proposed 3D face texture model. Specifically, a 3D face model is first recovered from a pair of frontal and profile face images, and then the 3D model is used to synthesize face images in novel views. Since there is only one face image for each subject in the PCSO background set (see Table 1), only the face images of 505 subjects from the FERET database are used as the target set.

Figure 11 shows multi-view face images synthesized for several subjects in the target set by using the proposed method. Face images generated for novel views using the proposed 3D face texture model appear to be visually realistic. Face verification using FaceVACS and densely sampled LBP is then performed with the enhanced gallery set with 8 synthesized views added to each subjects. As shown in Fig. 10, FaceVACS and LBP achieve significantly higher performance by using the enhanced gallery set. The verification rates of FaceVACS and LBP on the original gallery set at a False Alarm Rate (FAR) of $0.1 \%$ are merely $13.2 \%$ and $0.1 \%$, respectively. However, after representation capability enhancement with the proposed method, FaceVACS and LBP, respectively, achieve impressive $98.0 \%$ and $65.5 \%$ verification rates at a FAR of $0.1 \%$. These results further demonstrate the effectiveness of the proposed 3D texture model for improving 2D face recognition accuracy.

\section{Conclusions and Future Work}

Compared with a single 2D face image, a pair of frontal and profile face images provide more description of a subject's face. However, 3D face modeling from a pair of frontal and profile face images, captured at the booking time by law enforcement agencies, has received only limited attention. We have proposed a method for 3D face modeling 


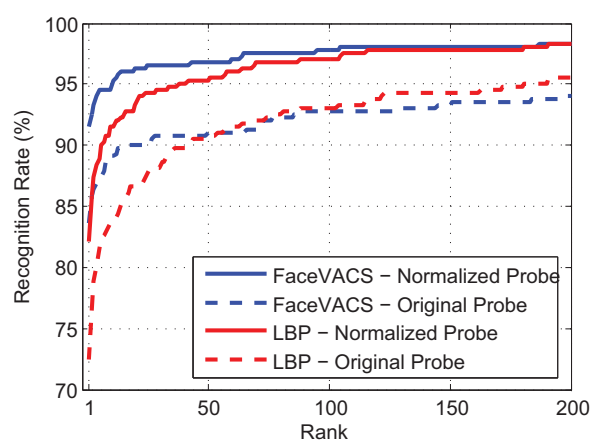

Figure 9. Face identification performance of FaceVACS and densely sampled LBP with and without using the proposed 3D face texture model to normalize the probe face images.

from uncalibrated frontal and profile face images. The correspondence between two uncalibrated face images is determined through landmark alignment. An initial 3D face shape is then reconstructed from the frontal face image followed by shape refinement based on the profile image. Face texture is extracted by mapping the frontal face image to the recovered 3D face shape. Although our method is proposed for building 3D face texture model from a pair of frontal and profile face images, it also works well with only a single input face image by bypassing the 3D shape refinement step in Section 3.4. Results of 2D face recognition experiments performed in two different scenarios shows that the proposed 3D face texture model is effective in improving the performance of a state-of-the-art commercial matcher. Future work includes automatic landmark detection for profile face images as well as texture extraction and fusion for the two input face images. We are also in the process of obtaining an operational database of profile and frontal face images acquired at the booking time.

\section{Acknowledgement}

The authors would like to thank Dr. Arun Ross for his suggestions. This research was supported by grants from the NSF Center for Identification Technology Research (CITeR).

\section{References}

[1] FaceVACS Software Developer Kit. Cognitec Systems GmbH, http://www.cognitec-systems.de, 2010.

[2] PittPatt Face Recognition SDK. Pittsburgh Pattern Recognition, http://www.pittpatt.com.

[3] USF DARPA HumanID 3D Face Database. Courtesy of Professor Sudeep Sarkar, University of South Florida.

[4] A. Ansari and M. A. Mottaleb. Automatic facial feature extraction and $3 \mathrm{~d}$ face modeling using two orthogonal views with application to 3d face recognition. Pattern Recognition, 38(12):2549-2563, 2005.

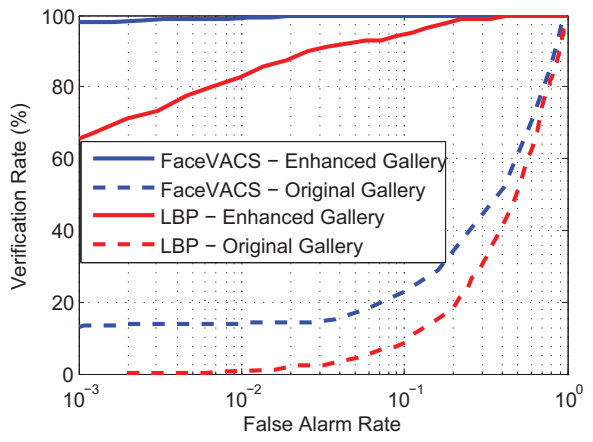

Figure 10. Face verification performance of FaceVACS and densely sampled LBP with and without using the proposed 3D face model to enhance the representation capability of gallery set.

[5] J. J. Atick, P. A. Griffin, and A. N. Redlich. Statistical approach to shape from shading: Reconstruction of threedimensional face surfaces from single two-dimensional images. Neural Computation, 8(6):1321-1340, 1996.

[6] R. Basri and D. W. Jacobs. Lambertian reflectance and linear subspaces. IEEE Trans. Pattern Analysis and Machine Intelligence, 25(2):218-233, 2003.

[7] D. Beymer and T. Poggio. Face recognition from one example view. In Proceedings of International Conference on Computer Vision, pages 500-507, 1995.

[8] V. Blanz and T. Vetter. Face recognition based on fitting a 3d morphable model. IEEE Trans. Pattern Analysis and Machine Intelligence, 25(9):1063-1074, 2003.

[9] M. Castelan and E. R. Hancock. Acquiring height data from a single image of a face using local shape indicators. Computer Vision and Image Understanding, 103(1):64-79, 2006.

[10] T. F. Cootes, D. Cooper, C. J. Taylor, and J. Graham. Active shape models - their training and application. Computer Vision and Image Understanding, 61(1):38-59, 1995.

[11] T. F. Cootes, G. J. Edwards, and C. J. Taylor. Active appearance models. IEEE Trans. Pattern Analysis and Machine Intelligence, 23(6):681-685, 2001.

[12] N. Faggian, A. Paplinski, and J. Sherrah. 3d morphable model fitting from multiple views. In Proceedings of $A u-$ tomatic Face and Gesture Recognition, pages 1-6, 2008.

[13] R. Gross and V. Brajovic. An image preprocessing algorithm for illumination invariant face recognition. In Proceedings of Audio and Video-Based Biometric Person Authentication, pages 10-18, 2003.

[14] H. Han, S. Shan, X. Chen, S. Lao, and W. Gao. Separability oriented preprocessing for illumination-invariant face recognition. In Proceedings of the 12th European Conference on Computer Vision, 2012 (To Appear).

[15] H. Han, S. Shan, L. Qing, X. Chen, and W. Gao. Lighting aware preprocessing for face recognition across varying illumination. In Proceedings of the 11th European Conference on Computer Vision, pages 308-321, 2010.

[16] J. Heo and M. Savvides. Rapid 3d face modeling using a frontal face and a profile face for accurate 2D pose synthesis. In Proceedings of Automatic Face and Gesture Recognition, pages 632-638, 2011. 

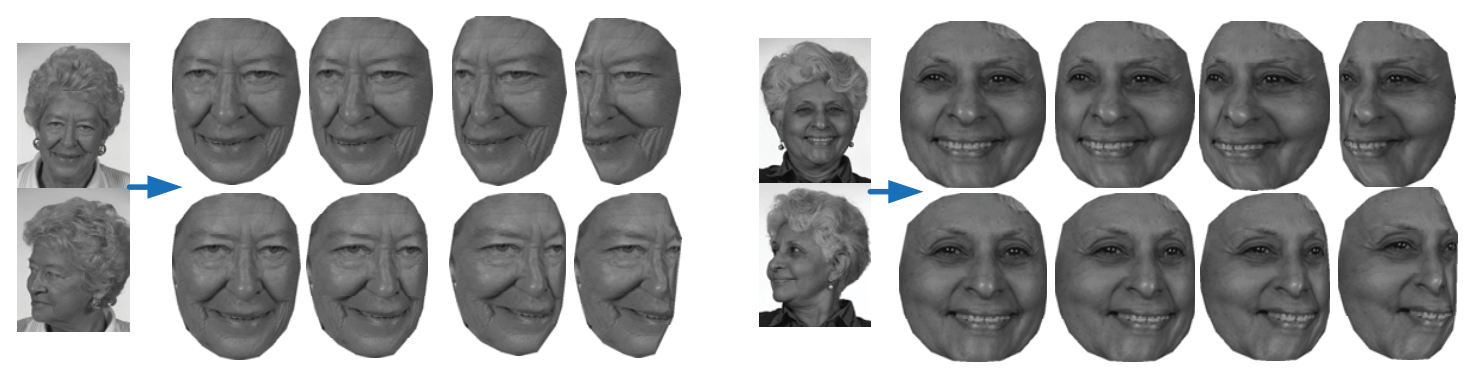

Figure 11. Example showing the capability of the proposed 3D face texture model to enhance the representation for two subjects in gallery set. The face model was constructed using a pair of frontal and profile face images of each subject.

[17] B. K. P. Horn and M. J. Brooks. Shape from Shading. MIT Press, 1989.

[18] H. H. S. Ip and L. Yin. Constructing a 3d head individualized model from two orthogonal views. The Visual Computer, 12(5):254-266, 1996.

[19] D. W. Jacobs, P. N. Belhumeur, and R. Basri. Comparing images under variable illumination. In Proceedings of IEEE Computer Vision and Pattern Recognition, pages 610-617, 1998.

[20] D. Jiang, Y. Hu, S. Yan, L. Zhang, H. Zhang, and W. Gao. Efficient $3 \mathrm{~d}$ reconstruction for face recognition. Pattern Recognition, 38(6):787-798, 2005.

[21] I. A. Kakadiaris, G. Passalis, G. Toderici, N. Murtuza, and T. Theoharis. $3 \mathrm{~d}$ face recognition. In Proceedings of British Machine Vision Conference, pages 869-878, 2006.

[22] S. Z. Li and A. K. Jain (eds.). Handbook of Face Recognition. Springer, 2nd edition, 2011

[23] S. Milborrow and F. Nicolls. Locating facial features with an extended active shape model. In Proceedings of European Conference on Computer Vision, pages 504-513, 2008.

[24] O. Ocegueda, G. Passalis, T. Theoharis, S. Shah, and I. A. Kakadiaris. UR3D-C: Linear dimensionality reduction for efficient 3d face recognition. In Proceedings of International Joint Conference on Biometrics, pages 1-6, 2011.

[25] T. Ojala, M. Pietikainen, and T. Maenpaa. Multiresolution gray-scale and rotation invariant texture classification with local binary patterns. IEEE Trans. Pattern Analysis and Machine Intelligence, 24(7):971-987, 2002.

[26] U. Park, Y. Tong, and A. K. Jain. Age-invariant face recognition. IEEE Trans. Pattern Analysis and Machine Intelligence, 32(5):947-954, 2010.

[27] P. J. Phillips, P. Grother, R. J. Micheals, D. M. Blackburn, E. Tabassi, and M. Bone. Face Recognition Vendor Test (FRVT). http://www.nist.gov/itl/iad/ig/frvt-2002.cfm.

[28] P. J. Phillips, S. A. R. H. Moon, and P. Rauss. The feret evaluation methodology for face recognition algorithms. IEEE Trans. Pattern Analysis and Machine Intelligence, 22(10):1090-1104, 2000.

[29] P. J. Phillips, W. T. Scruggs, A. J. O’Toole, P. J. Flynn, K. W. Bowyer, C. L. Schott, and M. Sharpe. FRVT 2006 and ICE 2006 large-scale experimental results. IEEE Trans. Pattern Analysis and Machine Intelligence, 32(5):831-846, 2010.

[30] A. Ross, K. Nandakumar, and A. K. Jain. Handbook of Multibiometrics. Springer, 2006.
[31] A. Shashua and T. Riklin-Raviv. The quotient image: Classbased re-rendering and recognition with varying illuminations. IEEE Trans. Pattern Analysis and Machine Intelligence, 23(2):129-139, 2001.

[32] T. Sim, S. Baker, and M. Bsat. The cmu pose, illumination, and expression database. IEEE Trans. Pattern Analysis and Machine Intelligence, 25(12):1615-1618, 2003.

[33] T. Sim and T. Kanade. Combining models and exemplars for face recognition: An illuminating example. In Proceedings of IEEE CVPR Workshop on Models versus Exemplars in Computer Vision, pages 1-10, 2001.

[34] X. Tan and B. Triggs. Enhanced local texture feature sets for face recognition under difficult lighting conditions. IEEE Trans. Image Processing, 19(6):1635-1650, 2010.

[35] M. Turk and A. Pentland. Eigenfaces for recognition. Journal of Cognitive Neuroscience, 3(1):71-86, 1991.

[36] T. Vetter. Synthesis of novel views from a single face image. International Journal of Computer Vision, 28(2):103116, 1998.

[37] T. Vetter and T. Poggio. Linear object classes and image synthesis from a single example image. IEEE Trans. Pattern Analysis and Machine Intelligence, 19(7):733-742, 1997.

[38] H. Wang and S. Z. Li. Face recognition under varying lighting conditions using self quotient image. In Proceedings of Automatic Face and Gesture Recognition, pages 498-505, 2004.

[39] L. Zhang and D. Samaras. Face recognition from a single training image under arbitrary unknown lighting using spherical harmonics. IEEE Trans. Pattern Analysis and Machine Intelligence, 28(3):351-363, 2006.

[40] X. Zhang, Y. Gao, and M. K. H. Leung. Recognizing rotated faces from frontal and side views: An approach toward effective use of mugshot databases. IEEE Trans. Information Forensics and Security, 3(4):684-697, 2008.

[41] M. Zhao, T. Chua, and T. Sim. Morphable face reconstruction with multiple images. In Proceedings of Automatic Face and Gesture Recognition, pages 597-602, 2006.

[42] W. Zhao and R. Chellappa. Illumination insensitive face recognition using symmetric shape from shading. In Proceedings of IEEE Computer Vision and Pattern Recognition, pages 286-293, 2000.

[43] W. Zhao and R. Chellappa. Symmetric shape-from-shading using self-ratio image. International Journal of Computer Vision, 45(1):55-75, 2001. 